\title{
A remark on Chen's theorem
}

\author{
by \\ YingChUn CAI (Shanghai)
}

1. Introduction. Let $p, p^{\prime}$ denote primes and $P_{2}$ denote an almost prime with at most two prime factors. For sufficiently large $x$ it is conjectured by Hardy and Littlewood [8] that

$$
\sum_{\substack{p \leq x \\ p+2=p^{\prime}}} 1=(1+o(1)) \frac{C x}{\log ^{2} x},
$$

where

$$
C=2 \prod_{p>2}\left(1-\frac{1}{(p-1)^{2}}\right) .
$$

This conjecture still remains open. The best result in this respect is due to Chen Jingrun [1] who showed in 1973 that

$$
\pi_{1,2}(x)>\frac{0.335 C x}{\log ^{2} x},
$$

where

$$
\pi_{1,2}(x)=\sum_{\substack{p \leq x \\ p+2=P_{2}}} 1 .
$$

The constant 0.335 was improved successively to $0.3445,0.3772,0.405,0.71$, 1.015, 1.05 by Halberstam [7], Chen Jingrun [2, 3], Fouvry and Grupp [4], H. Q. Liu [10] and J. Wu [12] respectively.

In this paper we obtain the following result.

THEOREM.

$$
\pi_{1,2}(x)>\frac{1.0974 C x}{\log ^{2} x} .
$$

2000 Mathematics Subject Classification: Primary 11N36.

Project supported by The National Natural Science Fundation of China (grant no. 19801021 and 10171060) and by MCSEC. 
2. Some lemmas. We denote by $\tau_{k}(n)$ the usual divisor function for $k \geq 2$ and $\tau_{2}(n)=\tau(n)$. For the definition of well-factorable functions we refer the reader to [4]. In the following we denote by $\lambda(q)$ a well-factorable function of level $Q$ and of order $k$.

Lemma 1 [4]. For an arithmetical function $\lambda^{\prime}$ of level $Q^{\prime}$ and of order $k^{\prime}, Q^{\prime} \leq Q, \lambda * \lambda^{\prime}$ is a well-factorable function of level $Q Q^{\prime}$ and of order $k+k^{\prime}$.

Let $\mathcal{A}$ denote a finite set of integers, $\mathcal{P}$ an infinite set of primes and $\overline{\mathcal{P}}$ the set of primes that do not belong to $\mathcal{P}$. Let $z \geq 2$. Put

$$
\begin{gathered}
P(z)=\prod_{p<z, p \in \mathcal{P}} p, \quad \mathcal{P}(q)=\{p \mid p \in \mathcal{P},(p, q)=1\} \\
S(\mathcal{A}, z)=S(\mathcal{A} ; \mathcal{P}, z)=\sum_{a \in \mathcal{A},(a, P(z))=1} 1, \quad \mathcal{A}_{d}=\{a \mid a \in \mathcal{A}, a \equiv 0(\bmod d)\} .
\end{gathered}
$$

Lemma 2 [9]. Let

$$
\begin{gathered}
\left|\mathcal{A}_{d}\right|=\frac{\omega(d)}{d} X+r_{d}, \quad \mu(d) \neq 0, \quad(d, \overline{\mathcal{P}})=1, \\
\frac{V\left(z_{1}\right)}{V\left(z_{2}\right)} \leq \frac{\log z_{2}}{\log z_{1}}\left(1+\frac{K_{1}}{\log z_{1}}\right), \quad K_{1}>1, \quad z_{2}>z_{1} \geq 2, \\
\sum_{\substack{z_{1} \leq p<z_{2} \\
p \in \bar{P}}} \sum_{\alpha \geq 2} \frac{\omega\left(p^{\alpha}\right)}{p^{\alpha}} \leq \frac{K_{2}}{\log 3 z_{1}}, \quad K_{2}>1,
\end{gathered}
$$

where $\omega(d)$ is a multiplicative function, $0 \leq \omega(p)<p, X>1$ is independent of $d$, and

$$
V(z)=\prod_{p \mid P(z)}\left(1-\frac{\omega(p)}{p}\right) .
$$

Then for $0<\varepsilon<10^{-5}, 2 \leq z \leq Q^{1 / 2}$, we have

$$
\begin{aligned}
& S(\mathcal{A}, \mathcal{P}, z) \geq X V(z)(f(s)-E)-\sum_{l<L} \sum_{q} \lambda_{l}^{-}(q) r(q), \\
& S(\mathcal{A}, \mathcal{P}, z) \leq X V(z)(F(s)+E)+\sum_{l<L} \sum_{q} \lambda_{l}^{+}(q) r(q),
\end{aligned}
$$

where $\lambda_{l}^{ \pm}$are well-factorable functions of level $Q$ and

$$
L=\exp \left(8 \varepsilon^{-3}\right), \quad E \ll \varepsilon+\varepsilon^{-8} \exp \left(K_{1}+K_{2}\right) \log ^{-1 / 3} Q,
$$




$$
s=\frac{\log Q}{\log z}, \quad\left|\lambda_{l}^{ \pm}(q)\right| \leq 1, \quad \lambda_{l}^{ \pm}(q)=0 \quad \text { for }(q, P(z))=1
$$

$f(s)$ and $F(s)$ are determined by the following differential-difference equation:

$$
\left\{\begin{array}{l}
F(s)=2 e^{\gamma} / s, \quad f(s)=0, \quad 0<s \leq 2, \\
(s F(s))^{\prime}=f(s-1), \quad(s f(s))^{\prime}=F(s-1), \quad s \geq 2 .
\end{array}\right.
$$

Here and below $\gamma$ is Euler's constant.

LEMMA 3 [7]. We have

$$
\begin{aligned}
& F(s)=\left\{\begin{array}{l}
\frac{2 e^{\gamma}}{s}, \quad 0<s \leq 3, \\
\frac{2 e^{\gamma}}{s}\left(1+\int_{2}^{s-1} \frac{\log (t-1)}{t} d t\right), \quad 3 \leq s \leq 5 ;
\end{array}\right. \\
& f(s)=\left\{\begin{array}{l}
\frac{2 e^{\gamma} \log (s-1)}{s}, \quad 2 \leq s \leq 4, \\
\frac{2 e^{\gamma}}{s}\left(\log (s-1)+\int_{3}^{s-1} \frac{d t}{t} \int_{2}^{t-1} \frac{\log (u-1)}{u} d u\right), \quad 4 \leq s \leq 6 .
\end{array}\right.
\end{aligned}
$$

Lemma 4 [4]. Let $Q=x^{4 / 7-\varepsilon}$ and $\varepsilon>0$. For any given $A>0$ and $|a| \leq \log ^{A} x$

$$
\sum_{(q, a)=1} \lambda(q)\left(\pi(x ; q, a)-\frac{\operatorname{Li} x}{\varphi(q)}\right)=O_{A, \varepsilon, a}\left(\frac{x}{\log ^{A} x}\right) .
$$

LEMMA 5 [4]. Let $\left(\alpha_{m}\right)$ and $\left(\beta_{n}\right)$ be two sequences satisfying the following conditions:

- $M \geq x^{\varepsilon}, \alpha_{m}=0$ for $m \notin[M, 2 M],\left|\alpha_{m}\right| \leq \tau_{k}(m)$;

- $N \geq x^{\varepsilon}, \beta_{n}=0$ for $n \notin[N, 2 N],\left|\beta_{n}\right| \leq \tau_{k}(n)$;

- for any given $e \geq 1, d \geq 1,(d, l)=1, A>0$,

$$
\sum_{\substack{n \equiv l(d) \\(n, e)=1}} \beta_{n}=\frac{1}{\varphi(d)} \sum_{(n, d e)=1} \beta_{n}+O\left(\frac{N \tau(e)^{B}}{\log ^{A} N}\right)
$$

- if $p \mid n \rightarrow p<\exp \left(\log ^{1 / 2} x\right)$ then $\beta_{n}=0$,

where $k$ and $B$ are constants. Let $M N \leq x, v=\log N / \log x, Q=x^{\theta(v)-2 \varepsilon}$ where $\theta(v)$ is defined by 


$$
\theta(v)= \begin{cases}(1+v) / 2, & 0 \leq v \leq 1 / 10 \\ (13+2 v) / 24, & 1 / 10 \leq v \leq 1 / 6 \\ (3+2 v) / 6, & 1 / 6 \leq v \leq 1 / 4 \\ (2-v) / 3, & 1 / 4 \leq v \leq 2 / 7 \\ (2+v) / 4, & 2 / 7 \leq v \leq 2 / 5 \\ 1-v, & 2 / 5 \leq v \leq 1 / 2 \\ 1 / 2, & 1 / 2 \leq v \leq 1\end{cases}
$$

Then for any $A>0$ and $|a| \leq \log ^{A} x$,

$$
\sum_{(q, a)=1} \lambda(q)\left(\sum_{m n \equiv a(q)} \alpha_{m} \beta_{n}-\frac{1}{\varphi(q)} \sum_{(m n, q)=1} \alpha_{m} \beta_{n}\right)=O_{A, \varepsilon, k, B}\left(\frac{x}{\log ^{A} x}\right) .
$$

LEMMA 6 [5]. Let $\xi(\cdot)$ denote an arithmetical function such that

$$
|\xi(q)| \leq \log x, \quad \xi(q)=0 \quad \text { for } q>Q_{1} .
$$

Then

$$
\sum_{\left(q q_{1}, a\right)=1} \lambda(q) \xi\left(q_{1}\right)\left(\pi\left(x ; q q_{1}, a\right)-\frac{\operatorname{Li} x}{\varphi\left(q q_{1}\right)}\right)=O_{A, \varepsilon, a}\left(\frac{x}{\log ^{A} x}\right)
$$

if either

$$
\begin{aligned}
& Q_{1} \leq Q, \quad Q_{1} Q \leq x^{4 / 7-\varepsilon}, \quad \text { or } \\
& Q_{1} \geq Q, \quad Q_{1}^{6} Q \leq x^{2-\varepsilon}, \quad \text { or } \\
& \xi(q)=\Lambda(q), \quad Q_{1} Q \leq x^{11 / 20-\varepsilon}, \quad Q_{1} \leq x^{1 / 3-\varepsilon} .
\end{aligned}
$$

LEMMA 7 [5]. Let $\eta>0$ and define

$$
g(t)= \begin{cases}4 / 7, & 0 \leq t \leq 2 / 7-\eta \\ 11 / 20, & 2 / 7-\eta \leq t \leq 1 / 3-\eta \\ 1 / 2, & 1 / 3-\eta \leq t \leq 1 / 2-\eta\end{cases}
$$

Then for any $A>0, \varepsilon>0$ and $|a| \leq \log ^{A} x$,

$$
\sum_{x^{t} \leq p<2 x^{t}} \sum_{(q, a)=1} \lambda(q)\left(\pi(x ; p q, a)-\frac{\operatorname{Li} x}{\varphi(p q)}\right)=O_{A, k, a}\left(\frac{x}{\log ^{A} x}\right)
$$

where $Q=x^{g(t)-t-\varepsilon}$.

Lemma 8 [11]. Let

$$
x>1, \quad z=x^{1 / u}, \quad Q(z)=\prod_{p<z} p .
$$


Then for $u \geq u_{0}>1$, we have

$$
\sum_{\substack{n \leq x \\(n, Q(z))=1}} 1=w(u) \frac{x}{\log z}+O\left(\frac{x}{\log ^{2} z}\right),
$$

where $w(u)$ is determined by the following differential-difference equation:

$$
\left\{\begin{array}{l}
w(u)=1 / u, \quad 1 \leq u \leq 2 \\
(u w(u))^{\prime}=w(u-1), \quad u \geq 2 .
\end{array}\right.
$$

Moreover,

$$
w(u)<\frac{1}{1.763} \quad \text { for } u \geq 2 .
$$

3. Weighted sieve method. Let $x$ be a sufficiently large real number and put

$$
\begin{aligned}
& \mathcal{A}=\{a \mid a=p+2, p \leq x\}, \\
& \mathcal{P}=\{p \mid(p, 2)=1\} .
\end{aligned}
$$

Lemma 9 [3]. Let $0<\alpha<\beta<1 / 3$ and $\alpha+3 \beta>1$. Then

$$
\begin{aligned}
\pi_{1,2}(x) \geq & \sum_{\substack{a \in \mathcal{A} \\
\left(a, 2 P\left(x^{\alpha}\right)\right)=1}}\left(1-\frac{1}{2} \varrho_{1}(a)-\frac{1}{2} \varrho_{2}(a)-\varrho_{3}(a)+\frac{1}{2} \varrho_{4}(a)\right)+O\left(x^{1-\alpha}\right) \\
\geq & S\left(\mathcal{A}, x^{\alpha}\right)-\frac{1}{2} \sum_{x^{\alpha} \leq p<x^{\beta}} S\left(\mathcal{A}_{p}, x^{\alpha}\right) \\
& -\frac{1}{2} \sum_{x^{\alpha} \leq p_{1}<x^{\beta} \leq p_{2}<\left(x / p_{1}\right)^{1 / 2}} S\left(\mathcal{A}_{p_{1} p_{2}}, p_{2}\right)-\sum_{x^{\beta} \leq p_{1}<p_{2}<\left(x / p_{1}\right)^{1 / 2}} S\left(\mathcal{A}_{\left.p_{1} p_{2}, p_{2}\right)} S\left(\mathcal{A}_{p_{1} p_{2} p_{3}} ; \mathcal{P}\left(p_{1}\right), p_{2}\right)+O\left(x^{1-\alpha}\right),\right.
\end{aligned}
$$

where

$$
\begin{aligned}
& \varrho_{1}(a)=\sum_{\substack{p \mid a \\
x^{\alpha} \leq p<x^{\beta}}} 1 ; \\
& \varrho_{2}(a)=\left\{\begin{array}{ll}
1, & a=p_{1} p_{2} p_{3}, \\
0, & \text { otherwise }
\end{array} x^{\alpha} \leq p_{1}<x^{\beta} \leq p_{2}<p_{3},\right. \\
& \varrho_{3}(a)=\left\{\begin{array}{ll}
1, & a=p_{1} p_{2} p_{3}, \\
0, & \text { otherwise }
\end{array} x^{\beta} \leq p_{1}<p_{2}<p_{3},\right. \\
& \varrho_{4}(a)= \begin{cases}1, & a=p_{1} p_{2} p_{3} n, x^{\alpha} \leq p_{1}<p_{2}<p_{3}<x^{\beta},\left(a, 2 p_{1}^{-1} P\left(p_{2}\right)\right)=1, \\
0, & \text { otherwise. }\end{cases}
\end{aligned}
$$


Proof. Since the second inequality can be deduced from the first one easily, it suffices to prove the first inequality. Let

$$
v_{2}(a)=\sum_{p^{m} \mid a} m, \quad \lambda(a)= \begin{cases}1, & v_{2}(a) \leq 2 \\ 0, & v_{2}(a)>2\end{cases}
$$

Then

$$
\pi_{1,2}(x) \geq \sum_{\substack{a \in \mathcal{A} \\\left(a, P\left(x^{\alpha}\right)\right)=1}} \lambda(a)=\sum_{\substack{a \in \mathcal{A} \\\left(a, P\left(x^{\alpha}\right)\right)=1}} \mu^{2}(a) \lambda(a)+O\left(x^{1-\alpha}\right) .
$$

On the other hand,

$$
\begin{aligned}
& \sum_{\substack{a \in \mathcal{A} \\
\left(a, P\left(x^{\alpha}\right)\right)=1}}\left(1-\frac{1}{2} \varrho_{1}(a)-\frac{1}{2} \varrho_{2}(a)-\varrho_{3}(a)+\frac{1}{2} \varrho_{4}(a)\right) \\
& =\sum_{\substack{a \in \mathcal{A} \\
\left(a, P\left(x^{\alpha}\right)\right)=1}} \mu^{2}(a)\left(1-\frac{1}{2} \varrho_{1}(a)-\frac{1}{2} \varrho_{2}(a)-\varrho_{3}(a)+\frac{1}{2} \varrho_{4}(a)\right)+O\left(x^{1-\alpha}\right) .
\end{aligned}
$$

For

$$
\mu^{2}(a)=1, \quad\left(a, P\left(x^{\alpha}\right)\right)=1,
$$

we have three cases:

1) $v_{2}(a) \leq 2$. Then $\varrho_{4}(a)=0$, and

$$
\lambda(a)=1 \geq 1-\frac{1}{2} \varrho_{1}(a)-\frac{1}{2} \varrho_{2}(a)-\varrho_{3}(a)+\frac{1}{2} \varrho_{4}(a) .
$$

2) $v_{2}(a)=3$. If $\varrho_{1}(a)=0$, then $\varrho_{3}(a)=1, \varrho_{2}(a)=\varrho_{4}(a)=0$, and

$$
\lambda(a)=0=1-\frac{1}{2} \varrho_{1}(a)-\frac{1}{2} \varrho_{2}(a)-\varrho_{3}(a)+\frac{1}{2} \varrho_{4}(a) .
$$

If $\varrho_{1}(a)=1$, then $\varrho_{3}(a)=\varrho_{4}(a)=0$, and $\varrho_{2}(a)=1$, hence

$$
\lambda(a)=0=1-\frac{1}{2} \varrho_{1}(a)-\frac{1}{2} \varrho_{2}(a)-\varrho_{3}(a)+\frac{1}{2} \varrho_{4}(a) .
$$

If $\varrho_{1}(a)=2$, then $\varrho_{2}(a)=\varrho_{3}(a)=\varrho_{4}(a)=0$, and

$$
\lambda(a)=0=1-\frac{1}{2} \varrho_{1}(a)-\frac{1}{2} \varrho_{2}(a)-\varrho_{3}(a)+\frac{1}{2} \varrho_{4}(a) .
$$

If $\varrho_{1}(a)=3$, then $\varrho_{2}(a)=\varrho_{3}(a)=0, \varrho_{4}(a)=1$, and

$$
\lambda(a)=0=1-\frac{1}{2} \varrho_{1}(a)-\frac{1}{2} \varrho_{2}(a)-\varrho_{3}(a)+\frac{1}{2} \varrho_{4}(a) .
$$

3) $v_{2}(a) \geq 4$. Then $\varrho_{1}(a) \geq 2$. If $\varrho_{1}(a)=2$, then $\varrho_{2}(a)=\varrho_{3}(a)=\varrho_{4}(a)$ $=0$, and

$$
\lambda(a)=0=1-\frac{1}{2} \varrho_{1}(a)-\frac{1}{2} \varrho_{2}(a)-\varrho_{3}(a)+\frac{1}{2} \varrho_{4}(a) .
$$


If $\varrho_{1}(a) \geq 3$, then $\varrho_{2}(a)=\varrho_{3}(a)=0, \varrho_{4}(a)=1$, and

$$
\lambda(a)=0 \geq 1-\frac{1}{2} \varrho_{1}(a)-\frac{1}{2} \varrho_{2}(a)-\varrho_{3}(a)+\frac{1}{2} \varrho_{4}(a) .
$$

Combining the above arguments we complete the proof of Lemma 9 .

LEMma 10. We have

$$
\begin{aligned}
& 2 \pi_{1,2}(x) \geq 2 S\left(\mathcal{A}, x^{1 / 10.5}\right)-\frac{1}{2} \sum_{x^{1 / 10.5} \leq p<x^{1 / 3.0015}} S\left(\mathcal{A}_{p}, x^{1 / 10.5}\right) \\
& -\frac{1}{2} \sum_{x^{1 / 10.5} \leq p_{1}<x^{1 / 3.0015} \leq p_{2}<\left(x / p_{1}\right)^{1 / 2}} S\left(\mathcal{A}_{p_{1} p_{2}}, p_{2}\right) \\
& -\sum_{x^{1 / 3.0015} \leq p_{1}<p_{2}<\left(x / p_{1}\right)^{1 / 2}} S\left(\mathcal{A}_{p_{1} p_{2}}, p_{2}\right) \\
& -\frac{1}{2} \sum_{x^{1 / 10.5} \leq p<x^{1 / 7.68}} S\left(\mathcal{A}_{p}, p\right)-\frac{1}{2} \sum_{x^{1 / 10.5} \leq p<x^{1 / 3.449}} S\left(\mathcal{A}_{p}, x^{1 / 10.5}\right) \\
& +\frac{1}{2} \sum_{x^{1 / 10.5} \leq p_{1}<p_{2}<x^{1 / 7.68}} S\left(\mathcal{A}_{p_{1} p_{2}}, x^{1 / 10.5}\right) \\
& +\frac{1}{2} \sum_{x^{1 / 10.5} \leq p_{1}<x^{1 / 7.68} \leq p_{2}<x^{8 / 21} p_{1}^{-1}} S\left(\mathcal{A}_{p_{1} p_{2}}, x^{1 / 10.5}\right) \\
& -\frac{1}{2} \sum_{x^{1 / 7.68} \leq p_{1}<x^{1 / 3.449} \leq p_{2}<\left(x / p_{1}\right)^{1 / 2}} S\left(\mathcal{A}_{p_{1} p_{2}}, p_{2}\right) \\
& -\sum_{x^{1 / 3.449} \leq p_{1}<p_{2}<\left(x / p_{1}\right)^{1 / 2}} S\left(\mathcal{A}_{p_{1} p_{2}}, p_{2}\right) \\
& -\frac{1}{2} \sum_{x^{1 / 10.5} \leq p_{1}<p_{2}<p_{3}<p_{4}<x^{1 / 7.68}} S\left(\mathcal{A}_{p_{1} p_{2} p_{3} p_{4}} ; \mathcal{P}\left(p_{1}\right), p_{2}\right) \\
& -\frac{1}{2} \sum_{x^{1 / 10.5} \leq p_{1}<p_{2}<p_{3}<x^{1 / 7.68} \leq p_{4}<x^{8 / 21} p_{3}^{-1}} S\left(\mathcal{A}_{p_{1} p_{2} p_{3} p_{4}} ; \mathcal{P}\left(p_{1}\right), p_{2}\right) \\
& +O\left(x^{9.5 / 10.5}\right) \\
& =2 \Sigma-\frac{1}{2} \Sigma_{1}-\frac{1}{2} \Sigma_{2}-\Sigma_{3}-\frac{1}{2} \Sigma_{4}-\frac{1}{2} \Sigma_{5}+\frac{1}{2} \Sigma_{6}+\frac{1}{2} \Sigma_{7} \\
& -\frac{1}{2} \Sigma_{8}-\Sigma_{9}-\frac{1}{2} \Sigma_{10}-\frac{1}{2} \Sigma_{11}+O\left(x^{9.5 / 10.5}\right) \text {. }
\end{aligned}
$$

Proof. By Buchstab's identity

$$
S\left(\mathcal{A}, z_{2}\right)=S\left(\mathcal{A}, z_{1}\right)-\sum_{z_{1} \leq p<z_{2}} S\left(\mathcal{A}_{p}, p\right), \quad 2 \leq z_{1} \leq z_{2},
$$


we have

$$
\begin{aligned}
S\left(\mathcal{A}, x^{1 / 7.68}\right)= & S\left(\mathcal{A}, x^{1 / 10.5}\right)-\frac{1}{2} \sum_{x^{1 / 10.5} \leq p<x^{1 / 7.68}} S\left(\mathcal{A}_{p}, p\right) \\
& -\frac{1}{2} \sum_{x^{1 / 10.5} \leq p<x^{1 / 7.68}} S\left(\mathcal{A}_{p}, x^{1 / 10.5}\right) \\
& +\frac{1}{2} \sum_{x^{1 / 10.5} \leq p_{1}<p_{2}<x^{1 / 7.68}} S\left(\mathcal{A}_{p_{1} p_{2}}, x^{1 / 10.5}\right) \\
& -\frac{1}{2} \sum_{x^{1 / 10.5} \leq p_{1}<p_{2}<p_{3}<x^{1 / 7.68}} S\left(\mathcal{A}_{p_{1} p_{2} p_{3}, p_{1}}\right),
\end{aligned}
$$

$$
\begin{aligned}
& \text { (3.4) } \sum_{x^{1 / 7.68} \leq p<x^{1 / 3.449}} S\left(\mathcal{A}_{p}, x^{1 / 7.68}\right) \\
& \leq \sum_{x^{1 / 7.68} \leq p<x^{2 / 7}} S\left(\mathcal{A}_{p}, x^{1 / 10.5}\right)
\end{aligned}
$$$$
-\sum_{x^{1 / 10.5} \leq p_{1}<x^{1 / 7.68} \leq p_{2}<x^{2 / 7}} S\left(\mathcal{A}_{p_{1} p_{2}}, p_{1}\right)+\sum_{x^{2 / 7} \leq p<x^{1 / 3.449}} S\left(\mathcal{A}_{p}, x^{1 / 10.5}\right)
$$$$
\leq \sum_{x^{1 / 7.68} \leq p<x^{1 / 3.449}} S\left(\mathcal{A}_{p}, x^{1 / 10.5}\right)-\sum_{x^{1 / 10.5} \leq p_{1}<x^{1 / 7.68} \leq p_{2}<x^{8 / 21} p_{1}^{-1}} S\left(\mathcal{A}_{\left.p_{1} p_{2}, p_{1}\right)}\right.
$$$$
=\sum_{x^{1 / 7.68} \leq p<x^{1 / 3.449}} S\left(\mathcal{A}_{p}, x^{1 / 10.5}\right)
$$$$
\sum_{x^{1 / 10.5} \leq p_{1}<x^{1 / 7.68} \leq p_{2}<x^{8 / 21} p_{1}^{-1}} S\left(\mathcal{A}_{p_{1} p_{2}}, x^{1 / 10.5}\right)
$$$$
+\sum_{x^{1 / 10.5} \leq p_{1}<p_{2}<x^{1 / 7.68} \leq p_{3}<x^{8 / 21} p_{2}^{-1}} S\left(\mathcal{A}_{p_{1} p_{2} p_{3}}, p_{1}\right)
$$

$$
\begin{gathered}
\sum_{x^{1 / 10.5} \leq p_{1}<p_{2}<p_{3}<x^{1 / 3.001}} S\left(\mathcal{A}_{p_{1} p_{2} p_{3}} ; \mathcal{P}\left(p_{1}\right), p_{2}\right) \\
-\sum_{x^{1 / 10.5} \leq p_{1}<p_{2}<p_{3}<x^{1 / 7.68}} S\left(\mathcal{A}_{p_{1} p_{2} p_{3}}, p_{1}\right)
\end{gathered}
$$$$
\sum_{x^{1 / 10.5} \leq p_{1}<p_{2}<x^{1 / 7.68} \leq p_{3}<x^{8 / 21} p_{2}^{-1}} S\left(\mathcal{A}_{p_{1} p_{2} p_{3}}, p_{1}\right)
$$

$$
\geq \sum_{x^{1 / 10.5} \leq p_{1}<p_{2}<p_{3}<x^{1 / 7.68}}\left(S\left(\mathcal{A}_{p_{1} p_{2} p_{3}} ; \mathcal{P}\left(p_{1}\right), p_{2}\right)-S\left(\mathcal{A}_{p_{1} p_{2} p_{3}}, p_{1}\right)\right)
$$




$$
\begin{aligned}
& +\sum_{x^{1 / 10.5} \leq p_{1}<p_{2}<x^{1 / 7.68} \leq p_{3}<x^{8 / 21} p_{2}^{-1}}\left(S\left(\mathcal{A}_{p_{1} p_{2} p_{3}} ; \mathcal{P}\left(p_{1}\right), p_{2}\right)-S\left(\mathcal{A}_{p_{1} p_{2} p_{3}, p_{1}}\right)\right) \\
& \geq-\sum_{x^{1 / 10.5} \leq p_{1}<p_{2}<p_{3}<p_{4}<x^{1 / 7.68}} S\left(\mathcal{A}_{p_{1} p_{2} p_{3} p_{4}} ; \mathcal{P}\left(p_{1}\right), p_{2}\right) \\
& -\sum_{x^{1 / 10.5} \leq p_{1}<p_{2}<p_{3}<x^{1 / 7.68} \leq p_{4}<x^{8 / 21}} S\left(\mathcal{A}_{3}^{-1} p_{p_{1} p_{3} p_{4}} ; \mathcal{P}\left(p_{1}\right), p_{2}\right) .
\end{aligned}
$$

By Lemma 9 with $(\alpha, \beta)=(1 / 10.5,1 / 3.0015)$ and $(\alpha, \beta)=(1 / 7.68,1 / 3.449)$ and (3.3)-(3.5), we complete the proof of Lemma 10.

4. Proof of the Theorem. In this section, the sets $\mathcal{A}$ and $\mathcal{P}$ are defined by (3.1) and (3.2) respectively. Let

For $(d, 2)=1$,

$$
X=\operatorname{Li} x \sim \frac{x}{\log x} .
$$

$$
r_{d}=\pi(x ; d,-2)-\frac{\operatorname{Li} x}{\varphi(d)}, \quad \omega(d)=\frac{d}{\varphi(d)}, \quad \mu(d) \neq 0 .
$$

1) Evaluation of $\Sigma, \Sigma_{4}, \Sigma_{6}, \Sigma_{7}$. Let $Q=x^{4 / 7-\varepsilon}$. By Mertens' theorem we have

$$
V(z)=\frac{e^{-\gamma} C}{\log z}\left(1+O\left(\frac{1}{\log z}\right)\right) .
$$

By Lemmas 2-4 and some routine arguments we get

$$
\begin{aligned}
\Sigma & \geq 3.5(1+O(\varepsilon)) \frac{C x}{\log ^{2} x}\left(\log 5+\int_{2}^{4} \frac{\log (s-1)}{s} \log \frac{5}{s+1} d s\right) \\
& \geq 5.8946937 \frac{C x}{\log ^{2} x} .
\end{aligned}
$$

Let $\lambda^{\prime}$ denote the characteristic function of the primes in the interval $\left[L, L^{\prime}\right)$, where $x^{1 / 10.5} \leq L<L^{\prime} \leq 2 L<x^{1 / 7.68}$ and $\lambda$ denote a well-factorable function of level $Q L^{-1}$. Then $L^{\prime}<Q L^{-1}$, by Lemma $1, \lambda * \lambda^{\prime}$ is a wellfactorable function of level $Q$. By Lemmas 2-4 and some routine arguments we get

$$
\begin{aligned}
\Sigma_{4} \leq & 3.5(1+O(\varepsilon)) \frac{C x}{\log ^{2} x}\left(\log \frac{35}{23.72}\left(1+\int_{2}^{16.72 / 7} \frac{\log (s-1)}{s} d s\right)\right. \\
& \left.+\int_{16.72 / 7}^{4} \frac{\log (s-1)}{s} \log \frac{5}{s+1} d s\right) \\
\leq & 1.61893 \frac{C x}{\log ^{2} x} .
\end{aligned}
$$


Let $\lambda_{1}^{\prime}, \lambda_{2}^{\prime}$ denote the characteristic functions of the primes in the intervals $\left[L_{1}, L_{1}^{\prime}\right)$ and $\left[L_{2}, L_{2}^{\prime}\right)$ respectively, where $x^{1 / 10.5+4 \varepsilon} \leq L_{1}<L_{1}^{\prime} \leq 2 L_{1}<$ $x^{1 / 7.68}, x^{1 / 7.68} \leq L_{2}<L_{2}^{\prime} \leq 2 L_{2}<x^{8 / 21}\left(2 L_{1}\right)^{-1}$, and $\lambda$ denote a wellfactorable function of level $Q\left(L_{1} L_{2}\right)^{-1}$. Then $L_{1}^{\prime}<Q\left(L_{1} L_{2}\right)^{-1}, L_{2}^{\prime}<Q L_{2}^{-1}$, by Lemma $1, \lambda * \lambda_{1}^{\prime}$ is a well-factorable function of level $Q L_{2}^{-1},\left(\lambda * \lambda_{1}^{\prime}\right) * \lambda_{2}^{\prime}$ is a well-factorable function of level $Q$. By Lemmas $2-4$ and some routine arguments we get

$$
\begin{aligned}
\Sigma_{7} \geq & 3.5(1+O(\varepsilon)) \\
& \times \frac{C x}{\log ^{2} x} \int_{1 / 10.5}^{1 / 7.68} \int_{1 / 7.68}^{8 / 21-t_{1}} \frac{\log \left(5-10.5\left(t_{1}+t_{2}\right)\right)}{t_{1} t_{2}\left(1-1.75\left(t_{1}+t_{2}\right)\right)} d t_{1} d t_{2} .
\end{aligned}
$$

Similarly,

$$
\begin{gathered}
\Sigma_{6} \geq 3.5(1+O(\varepsilon)) \frac{C x}{\log ^{2} x} \int_{1 / 10.5}^{1 / 7.68} \int_{t_{1}}^{1 / 7.68} \frac{\log \left(5-10.5\left(t_{1}+t_{2}\right)\right)}{t_{1} t_{2}\left(1-1.75\left(t_{1}+t_{2}\right)\right)} d t_{1} d t_{2}, \\
\Sigma_{6}+\Sigma_{7} \geq 1.188865 \frac{C x}{\log ^{2} x} .
\end{gathered}
$$

2) Evaluation of $\Sigma_{1}, \Sigma_{5}$. We have

$$
\begin{aligned}
\Sigma_{1} & =\left(\sum_{\substack{x^{1 / 10.5} \leq p<x^{2 / 7-\varepsilon} \\
x^{2 / 7-\varepsilon} \leq p<x^{0.29}}}+\sum_{x^{0.29} \leq p<x^{1 / 3.0015}}\right) S\left(\mathcal{A}_{p}, x^{1 / 10.5}\right) \\
& =S_{1}+S_{2}+S_{3}
\end{aligned}
$$

By Lemma 4 and the arguments used in [12], we get

$$
\begin{aligned}
S_{1} & \leq 3.5(1+O(\varepsilon)) \frac{C x}{\log ^{2} x}\left(\log 5+\int_{2}^{4} \frac{\log (s-1)}{s} \log \frac{5(5-s)}{s+1} d s\right) \\
& \leq 6.679727 \frac{C x}{\log ^{2} x} .
\end{aligned}
$$

By Lemmas 6 and 7 and the arguments used in [12], we have

$$
\begin{aligned}
& S_{2} \leq(1+O(\varepsilon)) \frac{C x}{\log ^{2} x} \log \frac{29}{26}, \\
& S_{3} \leq \frac{40 C x}{11 \log ^{2} x} \log \frac{52}{37.74785} .
\end{aligned}
$$

By (4.6)-(4.9) we get

(4.10) $\quad \Sigma_{1} \leq 3.5(1+O(\varepsilon)) \frac{C x}{\log ^{2} x}\left(\log 5+\int_{2}^{4} \frac{\log (s-1)}{s} \log \frac{5(5-s)}{s+1} d s\right)$ 


$$
\begin{aligned}
& +\frac{C x}{\log ^{2} x} \log \frac{29}{26}+\frac{40 C x}{11 \log ^{2} x} \log \frac{52}{37.7485} \\
\leq & 7.95371 \frac{C x}{\log ^{2} x} .
\end{aligned}
$$

Similarly,

$$
\begin{aligned}
\Sigma_{5} \leq & 3.5(1+O(\varepsilon)) \frac{C x}{\log ^{2} x}\left(\log 5+\int_{2}^{4} \frac{\log (s-1)}{s} \log \frac{5(5-s)}{s+1} d s\right) \\
& +\frac{C x}{\log ^{2} x} \log \frac{1}{0.898} \\
\leq & 6.78732 \frac{C x}{\log ^{2} x}
\end{aligned}
$$

3) Evaluation of $\Sigma_{10}, \Sigma_{11}$. We have

$$
\begin{aligned}
\Sigma_{10}= & \sum_{x^{1 / 10.5} \leq p_{1}<p_{2}<p_{3}<p_{4}<x^{1 / 7.68}} \sum_{\substack{a \in \mathcal{A}, p_{1} p_{2} p_{3} p_{4} \mid a \\
\left(a, p_{1}^{-1} P\left(p_{2}\right)\right)=1}} 1+O\left(x^{9.5 / 10.5}\right) \\
= & \sum_{x^{1 / 10.5} \leq p_{1}<p_{2}<p_{3}<p_{4}<x^{1 / 7.68}} \sum_{\substack{p=p_{1} p_{2} p_{3} p_{4} n-2 \\
1 \leq n \leq x / p_{1} p_{2} p_{3} p_{4},\left(n, p_{1}^{-1} P\left(p_{2}\right)\right)=1}} 1 \\
& +O\left(x^{9.5 / 10.5}\right) \\
= & S\left(\mathcal{B}, x^{1 / 2}\right)+O\left(x^{9.5 / 10.5}\right)
\end{aligned}
$$

where

$$
\begin{aligned}
\mathcal{B}=\left\{m p_{1} p_{2} p_{3} p_{4}-2 \mid x^{1 / 10.5}\right. & \leq p_{1}<p_{2}<p_{3}<p_{4}<x^{1 / 7.68} \\
& \left.m p_{1} p_{2} p_{3} p_{4} \leq x+2,\left(m, p_{1}^{-1} P\left(p_{2}\right)\right)=1\right\} .
\end{aligned}
$$

Applying the splitting technique used in [4] to remove the dependence of $m p_{4}$ on $p_{1}, p_{2}, p_{3}$, by Lemma 5 with $p_{1} p_{2} p_{3}$ for $n$, we get

$$
\begin{aligned}
& \sum_{(q, 2)=1} \lambda(q) \\
\times\left(\sum_{m p_{1} p_{2} p_{3} p_{4} \equiv 2(q)} \alpha_{m p_{4}} \beta_{p_{1} p_{2} p_{3}}-\frac{1}{\varphi(q)} \sum_{\left(m p_{1} p_{2} p_{3} p_{4}, q\right)}=1\right. & \left.\alpha_{m p_{4}} \beta_{p_{1} p_{2} p_{3}}\right) \\
& =O_{\varepsilon}\left(\frac{x}{\log ^{10} x}\right)
\end{aligned}
$$


with $Q=x^{4 / 7-\varepsilon}$. Lemmas 2 and 3 , when combined with (4.13), give (4.14) $S\left(\mathcal{B}, x^{1 / 2}\right)$

$$
\begin{aligned}
\leq & 3.5(1+O(\varepsilon)) \frac{C|\mathcal{B}|}{\log x} \\
= & 3.5(1+O(\varepsilon)) \frac{C}{\log x} \sum_{x^{1 / 10.5} \leq p_{1}<p_{2}<p_{3}<p_{4}<x^{1 / 7.68}} \sum_{\substack{1 \leq m \leq x /\left(p_{1} p_{2} p_{3} p_{4}\right) \\
\left(m, p_{1}^{-1} P\left(p_{2}\right)\right)=1}} 1 \\
& +O\left(x^{9.5 / 10.5}\right) \\
< & \frac{3.5 C}{1.763 \log x}(1+O(\varepsilon)) \sum_{x^{1 / 10.5} \leq p_{1}<p_{2}<p_{3}<p_{4}<x^{1 / 7.68}} \frac{x}{p_{1} p_{2} p_{3} p_{4} \log p_{2}} \\
& +O\left(x^{9.5 / 10.5}\right) \\
= & \frac{3.5 C x}{1.763 \log ^{2} x}(1+O(\varepsilon)) \int_{1 / 10.5}^{1 / 7.68} \frac{d t_{1}}{t_{1}} \int_{t_{1}}^{1 / 7.68} \frac{1}{t_{2}}\left(\frac{1}{t_{1}}-\frac{1}{t_{2}}\right) \log \frac{1}{7.68 t_{2}} d t_{2} .
\end{aligned}
$$

By (4.12) and (4.14) we get

$$
\begin{aligned}
\Sigma_{10} \leq & \frac{3.5}{1.763}(1+O(\varepsilon)) \frac{C x}{\log ^{2} x} \\
& \times \int_{1 / 10.5}^{1 / 7.68} \frac{d t_{1}}{t_{1}} \int_{t_{1}}^{1 / 7.68} \frac{1}{t_{2}}\left(\frac{1}{t_{1}}-\frac{1}{t_{2}}\right) \log \frac{1}{7.68 t_{2}} d t_{2} .
\end{aligned}
$$

By a similar method we get

$$
\text { (4.16) } \begin{aligned}
\Sigma_{11} \leq & \frac{3.5}{1.763}(1+O(\varepsilon)) \frac{C x}{\log ^{2} x} \\
& \times \int_{1 / 10.5}^{1 / 7.68} \frac{d t_{1}}{t_{1}} \int_{t_{1}}^{1 / 7.68} \frac{1}{t_{2}}\left(\frac{1}{t_{1}}-\frac{1}{t_{2}}\right) \log \left(7.68\left(\frac{8}{21}-t_{2}\right)\right) d t_{2} .
\end{aligned}
$$

By (4.15) and (4.16) we obtain

(4.17) $\quad \Sigma_{10}+\Sigma_{11}$

$$
\begin{aligned}
& \leq \frac{3.5}{1.763}(1+O(\varepsilon)) \frac{C x}{\log ^{2} x} \int_{1 / 10.5}^{1 / 7.68} \frac{d t_{1}}{t_{1}} \int_{t_{1}}^{1 / 7.68} \frac{1}{t_{2}}\left(\frac{1}{t_{1}}-\frac{1}{t_{2}}\right) \log \left(\frac{8}{21 t_{2}}-1\right) d t_{2} \\
& \leq 0.070549 \frac{C x}{\log ^{2} x} .
\end{aligned}
$$


4) Evaluation of $\Sigma_{2}, \Sigma_{3}, \Sigma_{8}, \Sigma_{9}$. By the arguments used in [10] we get

$$
\begin{aligned}
\Sigma_{2} \leq & 2(1+O(\varepsilon)) \frac{C x}{\log ^{2} x}\left(4 \int_{1 / 3.0015}^{2.0015 / 6.003} \frac{\log \frac{9.5-10.5 t}{2.0015-3.0015 t}}{t(2+t)(1-t)} d t\right. \\
& +4 \int_{2.0015 / 6.003}^{0.4} \frac{\log \frac{(9.5-10.5 t)(1-2 t)}{t}}{t(2+t)(1-t)} d t \\
& \left.+\int_{0.4}^{9.5 / 21} \frac{\log \frac{(9.5-10.5 t)(1-2 t)}{t}}{t(1-t)^{2}} d t\right) \\
\leq & 1.7711 \frac{C x}{\log ^{2} x},
\end{aligned}
$$

$$
\begin{aligned}
\Sigma_{3} & \leq 8(1+O(\varepsilon)) \frac{C x}{\log ^{2} x} \int_{1 / 3.0015}^{1 / 3} \frac{\log (1 / t-2)}{t(2+t)(1-t)} d t \\
& \leq 0.000003 \frac{C x}{\log ^{2} x},
\end{aligned}
$$

$$
\begin{aligned}
\Sigma_{8} \leq & 2(1+O(\varepsilon)) \frac{C x}{\log ^{2} x}\left(4 \int_{1 / 3.449}^{2.449 / 6.898} \frac{\log \frac{6.68-7.68 t}{2.449-3.449 t}}{t(2+t)(1-t)} d t\right. \\
& +4 \int_{2.449 / 6.898}^{0.4} \frac{\log \frac{(6.68-7.68 t)(1-2 t)}{t}}{t(2+t)(1-t)} d t \\
& \left.+\int_{0.4}^{6.68 / 15.36} \frac{\log \frac{(6.68-7.68 t)(1-2 t)}{t}}{t(1-t)^{2}} d t\right) \\
\leq & 1.90815 \frac{C x}{\log ^{2} x}
\end{aligned}
$$

$$
\Sigma_{9} \leq 8(1+O(\varepsilon)) \frac{C x}{\log ^{2} x} \int_{1 / 3.449}^{1 / 3} \frac{\log (1 / t-2)}{t(2+t)(1-t)} d t \leq 0.134048 \frac{C x}{\log ^{2} x} .
$$

Proof of the Theorem. By (4.2), (4.3), (4.5), (4.10), (4.11), (4.17)-(4.21), $2 \pi_{1,2}(x)$ is bounded below by

$$
\begin{aligned}
&(2 \cdot 5.89469-\frac{7.95372}{2}-\frac{1.7711}{2}-0.000003-\frac{1.61893}{2}-\frac{6.78732}{2} \\
&\left.+\frac{1.18886}{2}-\frac{1.90815}{2}-0.134048-\frac{0.070549}{2}\right) \frac{C x}{\log ^{2} x}>\frac{2.1948 C x}{\log ^{2} x}
\end{aligned}
$$


and so

$$
\pi_{1,2}(x)>\frac{1.0974 C x}{\log ^{2} x}
$$

The Theorem is proved.

\section{References}

[1] J. R. Chen, On the representation of a large even integer as the sum of a prime and the product of at most two primes, Sci. Sinica 16 (1973), 157-176.

[2] - On the representation of a large even integer as the sum of a prime and the product of at most two primes (II), ibid. 21 (1978), 421-430.

[3] - On the representation of a large even integer as the sum of a prime and the product of at most two primes (II), ibid., 477-494 (in Chinese).

[4] E. Fouvry and F. Grupp, On the switching principle in sieve theory, J. Reine Angew. Math. 370 (1986), 101-126.

[5] —, - Weighted sieves and twin prime type equations, Duke Math. J. 58 (1989), 731-748.

[6] H. Halberstam, A proof of Chen's Theorem, Astérisque 24-25 (1975), 281-293.

[7] H. Halberstam and H. E. Richert, Sieve Methods, Academic Press, London, 1974.

[8] G. H. Hardy and J. E. Littlewood, Some problems of 'partitio numerorum', III: On the expression of a number as a sum of primes, Acta Math. 44 (1923), 1-70.

[9] H. Iwaniec, A new form of the error term in the linear sieve, Acta Arith. 37 (1980), 307-320.

[10] H. Q. Liu, On prime twins problem, Sci. China Ser. A 33 (1990), 281-298.

[11] C. D. Pan and C. B. Pan, Goldbach Conjecture, Science Press, Beijing, 1992.

[12] J. Wu, Sur la suite des nombres premiers jumeaux, Acta Arith. 55 (1990), 365-394.

Department of Mathematics

Shanghai University

Shanghai 200436, P.R. China

E-mail: lumg0202@online.sh.cn

Received on 11.1.2001

and in revised form on 24.4.2001 